\title{
Emotion in Games
}

\author{
Georgios N.Yannakakis ${ }^{1}$, Kostas Karpouzis ${ }^{2}$, Ana Paiva ${ }^{3}$, and Eva Hudlicka ${ }^{4}$ \\ ${ }^{1}$ IT University of Copenhagen, Denmark \\ yannakakis@itu.dk \\ ${ }^{2}$ ICCS-NTUA, Greece \\ kkarpou@cs.ntua.gr \\ ${ }^{3}$ Instituto Superior Técnico/INESC-ID, Portugal \\ ana.paiva@inesc-id.pt \\ ${ }^{4}$ Psychometrix Associates, USA \\ Hudlicka@ieee.org
}

\begin{abstract}
Computer games are unique elicitors of emotion. Recognition of player emotion, dynamic construction of affective player models, and modelling emotions in non-playing characters, represent challenging areas of research and practice at the crossroads of cognitive and affective science, psychology, artificial intelligence and human-computer interaction. Techniques from AI and HCI can be used to recognize player affective states and to model emotion in non-playing characters. Multiple input modalities provide novel means for measuring player satisfaction and engagement. These data can then be used to adapt the gameplay to the player's state, to maximize player engagement and to close the affective game loop.

The Emotion in Games workshop (EmoGames $2011 \mathrm{http}: / /$ sirenproject. eu/content/acii-2011-workshop-emotion-games) will bring together researchers and practitioners in affective computing, user experience research, social psychology and cognition, machine learning, and $\mathrm{AI}$ and $\mathrm{HCI}$, to explore topics in player experience research, affect induction, sensing and modelling and affect-driven game adaptation, and modelling of emotion in non-playing characters. It will also provide new insights on how gaming can be used as a research platform, to induce and capture affective interactions with single and multiple users, and to model affect- and behaviour-related concepts, helping to operationalize concepts such as flow and engagement.

The workshop will include a keynote, paper and poster presentations, and panel discussions. Selected papers will appear in a special issue of the IEEE Transactions on Affective Computing, "Emotion in Games", in mid-2013.

The EmoGames2011 workshop is organized in coordination with the newly formed 'Emotion in Games' Special Interest Group (SIG) of the Humaine Association and the IEEE Computational Intelligence Society (CIS) Task Force on Player Satisfaction Modelling. We would like to thank all participants, as well as the members of the Program Committee, for their reviews of the workshop submissions: Elisabeth André, Ruth Aylett, Nadia Bianchi-Berthouze, Antonio Camurri, Marc Cavazza, Jonathan Gratch, Hatice Gunes, Dirk Heylen, Katherine Isbister, Stefanos Kollias, Maurizio Mancini, Anton Nijholt, Julian Togelius, Asimina Vasalou, Gualtiero Volpe, Catherine Pelachaud, and Tom Ziemke.
\end{abstract}

Keywords: emotion, games, affective gaming. 